\title{
Symptomatic anomalous left circumflex artery arising from the right coronary cusp
}

\author{
Haytham Allaham, Abdullah Mansour, Kul Aggarawal, Obai Abdullah \\ Department of Cardiology, University of Missouri, Columbia, MO, USA
}

Adv Interv Cardiol 2018; 14, 1 (51): 103-104 DOI: https://doi.org/10.5114/aic.2018.74364

The anomalous origin of the left circumflex artery as an independent branch from the right coronary cusp is considered a rare variation. In the variant, the left anterior descending artery, left circumflex artery and right coronary artery arise from three different discrete ostia. The anomalous left circumflex artery course passes posterior to the aortic root through the atrioventricular groove to supply the lateral wall of the left ventricle. Despite the usual benign and asymptomatic course, the clinical importance of this anomaly is evident from its association with sudden cardiac death, syncope, and arrhythmias as a manifestation of myocardial ischemia [1].

We report a case of a 70-year-old male patient who presented to the emergency department with generalized fatigue and exertional dyspnea of 2-month duration. Past medical was significant for hypertension and hyperlipidemia. The patient's blood pressure was managed with hydrochlorothiazide, hydralazine, lisinopril, and metoprolol prior to this presentation. Vitals on admission were significant for an elevated blood pressure of 180/112 mm Hg. Physical examination was otherwise unremarkable. Troponin levels were normal and an electrocardiogram (ECG) demonstrated minimal $T$ wave inversion in the inferior leads.

A low normal ejection fraction of $50 \%$ was observed on the echocardiogram with moderate left ventricular hypertrophy and asynchrony of the basal inferior wall. A technetium-99m sestamibi myocardial perfusion scan was performed and revealed a small, mild, reversible hypo-perfusion defect that involved the anteroapical and inferior basal regions. The study was followed by coronary artery angiography which revealed non-obstructive coronary artery disease (Figure 1). An incidental finding of an anomalous left circumflex artery originating from a separate ostium located at the right coronary cusp was noted during the study. The left circumflex artery had a completely separate origin from the right coronary artery. The medical therapy was optimized by adding amlodipine and increasing the metoprolol dose. He was followed at the cardiology clinic one month after discharge with a significant improvement in blood pressure (140/86 mm Hg), symptoms and overall functional status.

The anomalous origin of the left circumflex artery from the right coronary system was first described by Antopol and Kugel in 1933 and has an estimated frequency of $0.32-0.67 \%$ [2]. The anomaly may be classified according to the site of origin into different subtypes: left circumflex artery arising as a direct branch from the right coronary artery (RCA), a common right system ostium bifurcating into the left circumflex artery and RCA, and, as in our case, RCA and left circumflex artery originating from two separate orifices. Complications of this anomaly with myocardial ischemia resulting in sudden cardiac death are a result of the slit-like ostia, angling from the retroaortic course of the vessel or compression of the anomalous artery by a dilated aorta [3]. We propose the latter as a cause of our patient's chest discomfort and transient ischemia contributing to his stress test findings.

Our case report illustrates the impact of cardiac supply demand mismatch that may be present in hypertensive patients with an anomalous left circumflex artery and can become deadly if not properly managed. The report further highlights the improvement of overall functional status with adequate control of blood pressure in this group of patients.

\section{Conflict of interest}

The authors declare no conflict of interest.

Corresponding author:

Haytham Allaham, Department of Cardiology, University of Missouri, 1 Hospital Drive, 65201 Columbia, USA, phone: +1 6173066145,

e-mail: Allahamh@health.missouri.edu

Received: 29.09.2017, accepted: 10.11.2017. 

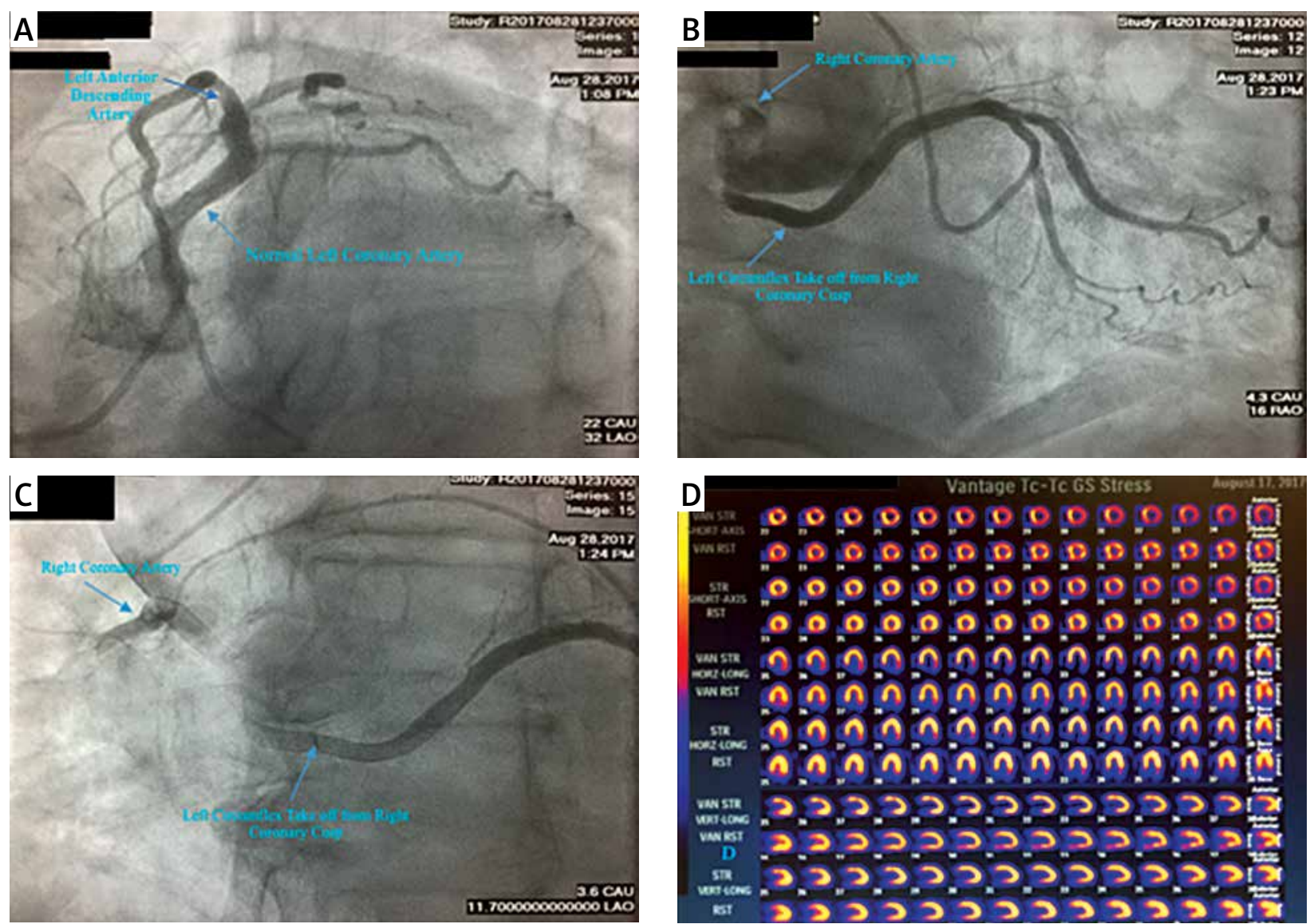

Figure 1. A - Coronary angiography showing normal left coronary artery arising from the left cusp, B, C - coronary angiography showing normal right coronary artery and anomalous take off of left circumflex from right coronary cusp, D - myocardial perfusion scan showing the anteroapical mild reversible hypoperfusion defect

\section{References}

1. Samarendra P, Kumari S, Hafeez M, et al. Anomalous circumflex coronary artery: benign or predisposed to selective atherosclerosis. Angiology 2001; 52: 521-6.

2. Antopol W, Kugel MA. Anamalous origin of the left circumflex coronary artery. Am Heart J 1933; 8: 802-6.

3. Aydin M, Ozeren A, Peksoy I, et al. Myocardial Ischemia caused by a coronary anomaly: left circumflex coronary artery arising from right sinus of valsalva. Tex Heart Inst J 2004; 31: 273-5. 